\title{
Carnets
}

Revue électronique d'études françaises de l'APEF

Deuxième série - 21 | 2021

Arts du vin

\section{Représentations bibliques de la vigne et du vin : bénédiction divine ou faute humaine?}

\section{Margarida Esperança Pina}

\section{(2) OpenEdition}

Journals

Édition électronique

URL : https://journals.openedition.org/carnets/12923

DOI : 10.4000/carnets. 12923

ISSN : 1646-7698

Éditeur

APEF

Référence électronique

Margarida Esperança Pina, «Représentations bibliques de la vigne et du vin : bénédiction divine ou faute humaine? », Carnets [En ligne], Deuxième série - 21 | 2021, mis en ligne le 31 mai 2021, consulté le 21 septembre 2021. URL : http://journals.openedition.org/carnets/12923 ; DOI : https://doi.org/ $10.4000 /$ carnets. 12923

Ce document a été généré automatiquement le 21 septembre 2021.

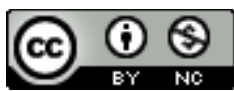

Carnets est mis à disposition selon les termes de la licence Creative Commons - Atribution - Pas d'utilisation commerciale 4.0 International. 


\title{
Représentations bibliques de la vigne et du vin : bénédiction divine ou faute humaine?
}

\author{
Margarida Pina
}

«Introduisez-moi dans la maison du vin » (Cantique

des Cantiques 2, 4)

1 L'imaginaire vinicole offre une multitude d'images autour de la vigne et du vin. Jouant un rôle essentiel dans le régime alimentaire et dans l'art de soigner, ce breuvage gagne une place très importante dans le contexte religieux. Les représentations sont nombreuses et vont d'un simple geste de bénédiction divine au plus complexe jeu du double sens, qu'il soit métaphorique ou allégorique, les images s'ouvrant sur le mystère de la relation de l'homme avec l'au-delà. L'imaginaire du vin emprunte, ainsi, à la culture christique une symbolique qui fait vivre in aeternum le pouvoir de la religion.

D'après J.H. Delgado (Delgado, 1985 : 56), le vin et le pain constituent l'alimentationbase du chrétien et représentent l'aliment spirituel essentiel à l'accomplissement du rituel de la communion. En quantité modérée, le vin alimente le corps, rend la santé, prévient les infirmités, aide à la digestion, équilibre la chaleur naturelle rend les idées plus claires, évite la tristesse et possède un don, celui de favoriser la procréation. En effet, au début de notre ère, la société ne considère pas évidente la relation entre l'acte de manger et l'acte de boire. Et plus tard, au Moyen Âge, la grande majorité des traités de morale faisant référence aux us et coutumes de table, n'évoquent que la boisson. Reconnaissant la qualité du vin, les commentaires sur la relation fatale entre le vin, les troubles sexuels et la luxure sont nombreux. Cependant, tout en étant néfaste pour la santé, ce délicieux breuvage sert un propos supérieur: mélangé avec de l'eau, le vin représente le Christ et l'eau, la progéniture humaine qui s'unit à Dieu dans le sacrement. Cette union sacrée se matérialise, incorporant une petite quantité d'eau au vin pour mettre en évidence la petitesse de l'homme face à Dieu. Ceci dit, le vin réunit plusieurs vertus : mélangé à de l'eau, il revigore le malade, associé à de l'huile, il soigne les blessures, combiné avec de la bile ou de la myrrhe, il anesthésie. Pour M.T. Lorcin, le 
vin, chaud et humide, reconstitue la chaleur du corps et des membres, élimine les mauvaises humeurs et rend la digestion plus légère (Lorcin, 1998 : 78).

3 En effet, Hippocrate (460 av. J.C. - 370 av. J.C.) fait déjà appel, dans sa pratique médicale, à un rééquilibrage de ces humeurs, dont le désordre provoque la maladie. D’après la médecine hippocratique, seul le régime alimentaire peut sauver le malade, en dosant d'une façon harmonieuse sec et humide, chaud et froid. Cette théorie certifie donc que l'eau est froide et humide, le vin sec et chaud.

4 Si nous reculons dans le temps, nous vérifions, par exemple, que dans le Chant IV de L'Odyssée, Homère (vIII ${ }^{\mathrm{e}}$ s. av. J.C.) prescrit le vin comme médication contre le chagrin : "Hélène verse dans la coupe de son époux une substance qui dissipe la tristesse, calme la colère et fait oublier tous les maux. » (Homère, $2018: 122)$

5 En ce qui concerne le début de notre ère, il faut prendre comme exemple la Palestine où la vigne, le raisin et le vin gagnent une autre dimension. C'est dire que leur importance dans la religion chrétienne est le vecteur primordial qui permettra, en parallèle avec l'essor du christianisme, le développement des plus grands vignobles. En toute évidence, les textes religieux se révèlent extrêmement riches. Dans l'aphorisme du talmud "Il n'y a pas de joie sans vin» ou dans cet autre "Quand le vin vient à manquer, les drogues deviennent nécessaires ", nous soulignons la valeur euphorique et analeptique du vin. Celui-ci demeure un élixir de jeunesse mais symbolise aussi une renaissance, le début d'un nouveau cycle. $\mathrm{Vu}$ comme un balsamique ayant les qualités thérapeutiques de l'huile, les deux sont présents dans la parabole du bon Samaritain : «il banda ses plaies en versant de l'huile et du vin " (Luc 10,34). Dans la logique hébraïque de la période biblique, le vin joue un rôle dans la thérapeutique médicale car les médecins utilisaient des substances animales ainsi que des plantes médicinales, telles que la mandragore. Mais pour les administrer, le malade buvait des infusions, des vins et appliquait des poudres, pommades ou collyres. Nous avons aussi pu observer que, dans le temps, le vin est utilisé, dans le contexte de la médecine hébraïque, lors de la circoncision. Pratiquée au huitième jour, le rabbin (le mohel, en hébreu) asperge la plaie chirurgicale d'un peu de vin, réputé hémostatique et antiseptique.

6 Ce breuvage médical, aux qualités soignantes et régénératives, s'ouvre à une variété d'interprétations dans le contexte biblique. La Bible, verset après verset, raconte ce nectar d'une finesse unique, en cédant la place à une variété de métaphores et allégories autour du raisin. Ainsi, c'est Noé qui offre à l'humanité la toute première vigne. Après le déluge, ce personnage biblique plante du raisin, pour ensuite en boire le vin. Belle métaphore de sa ténacité face à un monde qui se perd et qui, grâce à une stratégie biblique, mène Noé à protéger les hommes d'une fin apocalyptique. Il devient le travailleur premier d'un nouveau cycle exceptionnel. Il est, donc, le "prototype ", l'agriculteur qui apprend de la main de Dieu à s'occuper de sa vigne et à produire du vin. Ou mieux, Noé assure la survivance de l'humanité. Or, en raison des aux excès, il s'enivre et perd le contrôle :

Noé fut le premier cultivateur à planter de la vigne. Il but du vin, s'enivra et se déshabilla complètement à l'intérieur de sa tente. Cham, père de Canaan, vit son père tout nu et en avertit ses deux frères, qui étaient dehors. (Genèse, 9, 18-27)

7 De même, l'ivresse évoque la perte de contrôle de soi-même. Et cette absence d'équilibre se perpétue au long des récits bibliques, ne serait-ce que pour marquer la fragilité de l'humain, vis-à-vis de Dieu. Dans le passage de Loth, l'imposition du mal, du diabolique, s'avère visible à travers le vin. Voilà pourquoi les filles de Loth l'enivrent 
pour pouvoir coucher avec leur père, une à la fois, assurant la descendance, sous une forme maléfique :

Loth avait peur de rester à Soar ; il quitta la ville et alla vivre dans la montagne. Ses deux filles l'accompagnèrent; il s'installa avec elles dans une grotte. Un jour l'aînée dit à sa sœur : « Notre père est vieux et il n'y a pas d'homme dans la région pour nous épouser, comme cela se fait partout. Viens, nous allons enivrer notre père, puis nous passerons la nuit avec lui, pour lui donner des descendants. » (Genèse 19, 30-38)

En effet, les filles de Loth provoquent un coma alcoolique à leur père, en le faisant boire pour, ensuite, assurer la descendance. Et ailleurs, servant l'amour et la jouissance, dans le Cantique des cantiques, le vin sert d'invitation à la sexualité : «Mangez, mes amis, buvez, enivrez-vous d'amour. » (Cantique des cantiques 5, 1)

9 À propos de ces deux épisodes, Philon d'Alexandrie (ca 20 av. J.C. - 45), illustre philosophe et politicien du judaïsme alexandrin, explique la fonction du vin dans la logique biblique :

Considérons, à présent ce que pense [de l'ivresse] le législateur [Moïse] sublime et sage en toutes choses. En maints passages de sa Loi, en effet, il fait mention du vin et de la plante qui lui donne naissance : la vigne. Aux uns, il concède le droit d'en boire largement, aux autres il ne le permet pas.... Moïse considère le vin pur comme un symbole correspondant non à une réalité unique, mais à plusieurs : divagation, délire, inconscience absolue, désir insatiable, que l'on ne saurait combler ni apaiser, joie festive, allégresse à propos de tout, nudité, enfin, manifestée dans tout ce qui vient d'être évoqué, qui fut celle, dit-il de Noé dans son ivresse. Il est bien dit, assurément, que tels sont les effets du vin. Toutefois, beaucoup de personnes qui, par choix délibéré de la sobriété, s'abstiennent d'y toucher sont exposés aux mêmes effets; et l'on peut voir les uns délirer, divaguer, d'autres plongés dans une totale inconscience, d'autres jamais rassasiés, et toujours, faute de science, assoiffés de désirs sans fin, d'autres en revanche tout rayonnantes d'allégresse, et d'autres nus au sens propre. (...) La nudité, elle, a maintes causes : l'ignorance des contraires; l'innocence et la simplicité des mœurs, la vérité et son pouvoir de dévoiler ce qui était voilé dans l'ombre. (Cambronne, 2007 : 70)

10 Les réactions au vin sont, donc, diverses. Dans L'Apocalypse, par exemple, le vin est synonyme de prostitution et de séduction de l'idolâtrie, étant associé à la débauche et à l'immoralité : « Un deuxième ange suivit le premier en disant : 'Elle est tombée, elle est tombée la grande Babylone! Elle a fait boire à toutes les nations le vin de sa furieuse immoralité.'« (Apocalypse 14,8)

De ce fait, indépendamment du rôle que joue le vin dans la narrative biblique, il faut que le vin devienne symbole de la complétude, de la conquête de la «Terre de la Promesse ", comme explique Cambronne :

La tradition yahviste fait donc de la vigne l'un des fruits de la restauration de l'ordre cosmique et de la fécondité naturelle, l'un des signes, avec l'arc-en-ciel, de la nouvelle alliance entre Dieu et les hommes. Don de la seconde création, la vigne et la joie qu'elle procure, sont un lien entre les hommes et Dieu, comme l'exprime le vieil adage : quand on trouve du jus dans une grappe, on dit: ne la détruis pas car c'est une bénédiction (Is 65, 8). Mais l'ambivalence de ce don est immédiatement soulignée: l'ivresse de Noé démontre combien l'homme doit être mesuré dans l'usage qu'il fait de la vigne. Lors de la vie au désert, durant l'Exode, la vigne ne cesse d'être un bien ardemment désiré par tout le peuple hébreu. Elle est, à ses yeux, le vrai symbole de la Terre de la Promesse, pas encore atteinte. (Cambronne, $2007: 57)$ 
11 À vrai dire, de nos jours, le souvenir de l'Exode est encore vécu par la tradition judaïque, lors du Séder de Pessah. Il s'agit d'un repas pascal juif au cours duquel est faite la lecture de textes bibliques. Les participants boivent du vin et consomment des aliments spécifiques pendant que des chants sont entonnés. Et pour revivre le commandement biblique, le Séder a lieu après la tombée de la nuit, lors du premier soir de Pessah pour commémorer l'Exode miraculeux de l'Égypte. Les étapes et les aliments se succèdent au long de la soirée mais nous souhaiterions souligner ici le rôle joué par le vin. Les convives ingèrent $\mathrm{du}$ vin car celui-ci représente la joie et le bonheur. Au nombre de quatre, chaque verre perpétue, dans le temps, le courage du peuple hébreu qui maintient les noms et la langue hébraïque, la haute moralité et la fidélité les uns aux autres.

De ce fait, dans la tradition biblique, le vin est symbole de prospérité et d'espoir. Le peuple juif a un seul désir : celui de trouver une terre fertile pour pouvoir, finalement, vivre en paix. Voilà le seul souhait que les Juifs adressent à Yahvé. Donc, à la veille de l'avènement de la Jérusalem nouvelle, c'est dans la représentation de cette vigne que se manifeste l'espérance messianique juive. Nous revenons, donc, à l'image de Noé, en tant que créateur de la vigne, cette vigne métaphorique qui permet au peuple d'Israël d'avoir confiance en un futur prospère pour les élus de Dieu.

D'ailleurs, nous ne devons pas nous fixer à peine sur les images de la vigne et du vin, au sens métaphorique. En fait, le paysage viticole parsème toutes les civilisations du Proche et Moyen-Orient et de la Méditerranée, depuis l'Antiquité. D'après les fouilles archéologiques, il est possible de confirmer que la consommation de vin provenant de raisins sauvages avait lieu dans le paléolithique. Il faut par ailleurs souligner que le début de la vigne cultivée au néolithique prouve que la culture viticole existe depuis très longtemps, notamment au nord des monts Zagros, en Iran, en Géorgie et au nordest de la Turquie, ayant gagné (à partir des terres du Caucase, des monts Zagros et du Taurus), petit à petit, le Proche-Orient, la Syrie et la Palestine. Voici pourquoi nous retrouvons, dans le contexte biblique, des descriptions des vignes et du vin dans le Caucase, sur le mont Ararat lors de la description de l'ivresse de Noé.

Sans revenir aux diverses références à la vigne et au vin comme faisant partie du régime alimentaire, rappelons principalement qu'elles sont liées aussi bien à un tableau positif - la vigne comme signe de richesse et de bénédiction divine - comme à un tableau négatif - l'ivresse comme un manque. Soulignons, tout d'abord, que dans les Saintes Écritures, la vie se programme à partir d'un rythme cyclique, d'après la loi :

L'Éternel parla à Moïse sur la montagne de Sinaï, et dit : parle aux enfants d'Israël, et tu leur diras : quand vous serez entrés dans le pays que je vous donne, la terre se reposera: ce sera un sabbat en l'honneur de l'Éternel. Pendant six années tu ensemenceras ton champ, pendant six années tu tailleras ta vigne; et tu en recueilleras le produit. Mais la septième année sera un sabbat, un temps de repos pour la terre, un sabbat en l'honneur de l'éternel : tu n'ensemenceras point ton champ, et tu ne tailleras point ta vigne. Tu ne moissonneras point ce qui proviendra des grains tombés de ta moisson, et tu ne vendangeras point les raisins de ta vigne non taillée : ce sera une année de repos pour la terre. (Lévitique 25, 1-5)

Dieu oblige, ainsi, l'homme à se reposer pendant un an, après avoir travaillé la terre pendant six ans. En imposant un interdit, Dieu prépare l'humanité pour une nouvelle vie. Cette métaphore du temps stagné, renforcée constamment par l'utilisation de la forme négative dans le discours (au niveau de la syntaxe) traduit, en toute évidence, 
l'intention de suspendre le temps humain. Cette image est, tout de reste, reprise maintes fois :

Quand vous ferez la moisson dans votre pays, tu laisseras un coin de ton champ sans le moissonner, et tu ne ramasseras pas ce qui reste à glaner. Tu ne cueilleras pas non plus les grappes restées dans ta vigne, et tu ne ramasseras pas les grains qui en seront tombés. Tu abandonneras cela au pauvre et à l'étranger. Je suis l'Éternel, votre Dieu. (Lévitique 19, 9-10)

Par ailleurs, le vin est aussi le symbole de la bénédiction de Dieu, figurant l'alliance entre Dieu et son peuple. De ce fait, du vin est offert à Dieu sur l'autel, lors de sacrifices: "Avec un dixième de fleur de farine pétrie dans un quart d'hin d'huile [d'olives] broyées, et une libation d'un quart d'hin de vin, pour un agneau. » (Exode 29, 40). Synonyme de bonheur, le vin est évoqué comme forme de célébration, lors du banquet avec Dieu, à la fin des temps : "Sur le mont Sion, le Seigneur de l'univers offrira à tous les peuples un banquet de viandes grasses arrosé de vins fins, des viandes tendres et grasses, des vins fins bien clarifiés. » (Isaïe 25, 6)

Ce même signe de richesse et de bénédiction divine se repend un peu partout dans la Bible, comme dans les versets «Que Dieu te donne de la rosée des cieux, et des ressources de la terre, et une abondance de blé et de vin nouveau »! (Genèse 27, 28) ou dans les versets «Et l'Éternel des armées fera, sur cette montagne, à tous les peuples, un festin de mets savoureux, un festin de vins vieux, de choses grasses pleines de moelle, de vins vieux bien décantés. » (Isaïe 25, 6).

qui concerne la joie de vivre en Dieu, celle-ci se perd et la misère s'annonce quand le peuple n'a pas de vin à boire. L'espoir disparaît, alors, et le chant de lamentation prophétique annonce l'affliction, les larmes et l'absence de vin (signe de réjouissance) :

Le vin nouveau est en deuil, la vigne dépérit; tous ceux qui étaient joyeux de cœur soupirent. La joie des tambourins a cessé, le bruit tumultueux de ceux qui se réjouissent a pris fin, la joie de la harpe a cessé. Ils ne boivent pas le vin en chantant, les boissons fortes sont amères pour ceux qui les boivent. La cité désertique est en ruine; toute maison est fermée, de sorte que personne n'y entre. Il y a un cri dans les rues au sujet du vin. Toute joie est assombrie, l'allégresse est bannie du pays. Il ne reste que dévastation dans la ville et la porte est brisée une ruine. (Isaïe $24,1-12$ )

Il en va de même pour cette allégorie nuptiale dans laquelle Israël est comparé à une épouse infidèle, oublieuse des bienfaits, selon une thématique chère au prophète Osée :

Accusez votre mère, accusez ! Car elle n'est pas ma femme et je ne suis pas son mari. Et qu'elle ôte ses prostitutions de devant sa face et ses adultères d'entre ses seins, de peur que je ne la déshabille entièrement, et que je ne la place là comme au jour de sa naissance, et que je n'en fasse comme un désert, et que je ne la rende comme une terre aride, et que je ne la fasse mourir de soif. Et je ne ferai pas miséricorde à ses enfants, car ce sont des enfants de prostitution. Car leur mère s'est prostituée, celle qui les a conçus s'est déshonorée. Car elle a dit : Je veux aller après mes amants qui m'ont donné mon pain et mon eau, ma laine et mon lin, mon huile et ma boisson. C'est pourquoi, voici, je vais fermer ton chemin avec des ronces et j'élèverai une clôture. Et elle ne trouvera pas ses sentiers. Et elle courra après ses amants et ne les atteindra pas, et elle les cherchera et ne les trouvera pas. Et elle dira : Je veux aller et je veux retourner vers mon premier mari, car c'était alors meilleur pour moi que maintenant. Et elle ne sait pas que c'est moi qui lui ai donné le blé, et le vin nouveau, et l'huile. Je lui ai multiplié aussi l'argent et l'or ; ils l'ont employé pour Baal. C'est pourquoi je reviendrai prendre mon blé en son temps et mon vin nouveau dans sa saison. Et j'ôterai ma laine et mon lin, qui devaient couvrir sa nudité. Et maintenant, je découvrirai sa honte aux yeux de ses amants et 
personne ne la délivrera de ma main. Et je ferai cesser toute sa joie, sa fête, sa nouvelle lune, et son sabbat, et toutes ses assemblées. Et je détruirai sa vigne et son figuier, dont elle disait: Ce sont là mes salaires que mes amants m'ont donnés. Et j'en ferai une forêt, et les bêtes des champs les dévoreront. (Osée 2,1-14) la vigne et de boire du vin fait naître l'espoir :

Voici, les jours viennent - déclare l'Éternel - où celui qui laboure suivra de près celui qui moissonne, et celui qui foule le raisin [suivra de près] celui qui répand la semence. Et les montagnes feront couler le jus de raisin et toutes les collines ruisselleront. Et je rétablirai les déportés de mon peuple Israël, et ils reconstruiront les villes dévastées et $\mathrm{y}$ habiteront, et ils planteront des vignes et en boiront le vin, et ils feront des jardins et en mangeront le fruit. (Amos 9, 13-15)

Dans les textes bibliques, en ce qui concerne l'hommage à Dieu, nous pouvons considérer deux types de culte : l'offrande ou l'abstention de vin (le mot "nâziréat ", transcrit de l'hébreu, signifie "séparation » et désigne une consécration qui peut être temporaire ou définitive.) D'un côté, l'offrande liturgique s'annonce comme un épisode qui sacralise, d'un point de vue symbolique, l'avenir. La rencontre entre Abraham et Melchisédech peut être vue, en effet, comme une vision: "Et Melchisédech, roi de Salem, fit apporter du pain et du vin - or il était sacrificateur du Dieu Très-Haut - et il le bénit et dit: Béni soit Abram par le Dieu Très-Haut, possesseur des cieux et de la terre. » (Genèse 14, 18-19). D'un autre côté, cette offrande gagne une valeur rituelle comme dans l'exemple qui suit : « Voici les offrandes de vin qu'on doit répandre pour chaque victime. » (Nombres 28, 14)

En ce qui concerne l'abstention de vin, la Bible fournit beaucoup d'exemples autour de l'interdit alimentaire, souvent réservé aux êtres d'exception (le Nazir). C'est le cas de la naissance extraordinaire de Samson :

Or il y avait un homme de Tsorha, de la famille des Danites, et son nom était Manoah. Et sa femme était stérile et n'avait pas d'enfants. Et l'Ange de l'Éternel apparut à la femme, et il lui dit : «Voici, tu es stérile et tu n'as pas d'enfants. Mais tu concevras et tu donneras naissance à un fils. Et maintenant, prends garde, je te prie, et ne bois ni vin ni boisson forte, et ne mange rien d'impur. Car voici, tu concevras, et tu donneras naissance à un fils, et le rasoir ne passera pas sur sa tête, car le jeune garçon sera naziréen de Dieu dès le ventre [de sa mère]. Et ce sera lui qui commencera à sauver Israël de la main des Philistins. (Juges 13, 2-5)

Voyons, à présent, comment est exalté, dans la Bible, l'amour de Dieu. Célébrer Dieu, c'est établir des comparaisons et le vin, dans cette logique, est une référence d'excellence parce qu'il permet de dire et comparer. Dans le Cantique des cantiques, l'amour et la fête sont annoncés grâce au double sens que les comparaisons et les métaphores offrent, comme dans les versets : «Que vos mamelles sont belles, ma sœur, mon épouse ! Vos mamelles sont plus belles que le vin, et l'odeur de vos parfums passe celle de tous les aromates. » (Cantique des cantiques 4,10 ) ou dans ces autres versets : "Prenez-nous les petits renards qui détruisent les vignes, car notre vigne est en fleur. » (Cantique des cantiques 15).

24 Au-delà des comparaisons qui font appel à la symbolique du vin, il faut réfléchir à toutes les allégories que les Saintes Écritures proposent. Dans le Livre de la Genèse, nous avons accès à une allégorie contenue dans un rêve que raconte le grand échanson à Joseph : 
" Il me semblait que je voyais devant moi un cep de vigne, où il y avait trois provins qui poussaient peu à peu, premièrement des boutons, ensuite des fleurs, et à la fin des raisins mûrs; et qu'ayant dans la main la coupe de Pharaon, j'ai pris cette grappe de raisin, je les ai pressés dans la coupe que je tenais, et en ai donné à boire au roi. " Joseph lui dit : «Voici l'interprétation de votre songe : les trois provins de la vigne marquent trois jours, après lesquels Pharaon se souviendra du service que vous lui rendiez: il vous rétablira dans votre première charge, et vous lui présenterez à boire, selon que vous aviez accoutumé de le faire auparavant dans le rang que vous teniez: il vous rétablira dans votre première charge, et vous lui présenterez à boire, selon que vous aviez accoutumé de le faire auparavant dans le rang que vous teniez. (Genèse 40, 9-15)

Les paraboles les plus utilisées dans l'analyse de la doctrine chrétienne sont placées dans les Évangiles et, tout en étant des récits brefs, elles fournissent un contenu allégorique, toujours moralisant. Et surtout, le récit bref permet la codification d'un message qui passe très rapidement au contenu souhaité. Dans le Nouveau Testament, d'une façon générale, le vin est utilisé dans les fêtes, comme motif de célébration. Par exemple, dans les noces de Cana, la valeur sacramentelle est établie grâce à la symbolique du vin. Jésus choisit la célébration d'un mariage pour se faire connaitre comme un signe messianique. C'est le scénario parfait car c'est l'espace de la joie, de la fête, le vin invitant à la fête. Et Jésus est ce vin nouveau. C'est, donc, un signe qui ouvre la voie pour l'Eucharistie, cet espace du divin :

Et le troisième jour, il y eut une noce à Cana en Galilée ; et la mère de Jésus était là. Et Jésus fut aussi invité à la noce, ainsi que ses disciples. Et le vin étant venu à manquer, la mère de Jésus lui dit: Ils n'ont pas de vin. [Et] Jésus lui dit: Qu'y a-t-il entre moi et toi, femme? Mon heure n'est pas encore venue. Sa mère dit aux serviteurs : Faites tout ce qu'il vous dira. Or il y avait là six jarres de pierre pour contenir de l'eau, placées là selon [l'usage de] la purification des Juifs, pouvant recevoir chacune deux ou trois mesures, Jésus leur dit : Remplissez d'eau les jarres ! Et ils les remplirent jusqu'en haut. Et il leur dit : Puisez maintenant et apportez-en au maître d'hôtel ! Et ils lui en apportèrent. Mais lorsque le maître d'hôtel eut goûté l'eau qui était devenue du vin - or il ne savait pas d'où venait celui-ci, mais les serviteurs qui avaient puisé l'eau le savaient - le maître d'hôtel appelle le marié et lui dit: Tout homme sert le bon vin en premier et puis le moindre, après qu'on a bien bu. Toi, tu as gardé le bon vin jusqu'à maintenant. Jésus fit ce commencement de [ses] miracles à Cana en Galilée. Et il manifesta sa gloire et ses disciples crurent en lui. (Jean 2,1-11)

De même, dans l'Évangile, le vin nouveau qu'il faut mettre dans de nouvelles outres est une préannonce de la Bonne Nouvelle comme proclame Jean : «Je suis la vraie vigne et mon Père est le vigneron. » (Jean 15, 1-15) ou dans l'Évangile de Mathieu : «On ne met pas non plus du vin nouveau dans de vieilles outres; autrement, les outres se rompent, le vin se répand, et les outres sont perdues ; mais on met le vin nouveau dans des outres neuves, et le vin et les outres se conservent. » (Mathieu 9, 17)

Indubitablement, c'est ce vin nouveau qui nous conduit à l'épisode le plus touchant. Lors de son dernier repas, Jésus-Christ annonce à ses disciples : «'Je suis le pain' telle quelle la Révélation faite à Moïse quand Dieu lui annonce : 'JE SUIS celui que je suis.' Et il dit : 'Tu diras ainsi aux fils d'Israël : JE SUIS m'a envoyé vers vous.' (Exode 3, 14). Et cette révélation se fait en présence du pain et de la coupe de vin :

En vérité, en vérité, je vous [le] dis : Celui qui croit [en moi] à la vie éternelle. Moi, je suis le pain de vie. Vos pères ont mangé la manne dans le désert, et ils sont morts. C'est là le pain qui descend du ciel, afin que celui qui en mange ne meure pas. Moi, je suis le pain vivant qui est descendu du ciel. Si quelqu'un mange de ce 
pain, il vivra éternellement. Or le pain que moi, je donnerai, c'est ma chair, [que je donnerai] pour la vie du monde. »

Alors les Juifs discutaient vivement entre eux, en disant : « Comment celui-ci peut-il nous donner [sa] chair à manger? " Jésus donc leur dit : «En vérité, en vérité, je vous [le] dis : Si vous ne mangez pas la chair du Fils de l'homme et ne buvez pas son sang, vous n'avez pas la vie en vous-mêmes. Celui qui se nourrit de ma chair et qui boit mon sang a la vie éternelle et moi, je le ressusciterai au dernier jour. Car ma chair est vraiment une nourriture et mon sang est vraiment une boisson. Celui qui se nourrit de ma chair et qui boit mon sang demeure en moi et moi en lui. Comme le Père [qui est] vivant m'a envoyé, et que moi, je vis à cause du Père, de même celui qui se nourrit de moi, celui-là aussi vivra à cause de moi. Alors les Juifs discutaient vivement entre eux, en disant : «Comment celui-ci peut-il nous donner [sa] chair à manger? " Jésus donc leur dit: «En vérité, en vérité, je vous [le] dis: Si vous ne mangez pas la chair du Fils de l'homme et ne buvez pas son sang, vous n'avez pas la vie en vous-mêmes. Celui qui se nourrit de ma chair et qui boit mon sang a la vie éternelle et moi, je le ressusciterai au dernier jour. Car ma chair est vraiment une nourriture et mon sang est vraiment une boisson. Celui qui se nourrit de ma chair et qui boit mon sang demeure en moi et moi en lui. Comme le Père [qui est] vivant m'a envoyé, et que moi, je vis à cause du Père, de même celui qui se nourrit de moi, celui-là aussi vivra à cause de moi. (Jean 6, 47-57)$$
\begin{aligned}
& \text { percevons l'essence de la liturgie eucharistique, au sein de laquelle la consécration } \\
& \text { un double rôle. Il s'agit, en même temps, d'un souvenir et d'un engagement } \\
& \text { légitime, à chaque fois, la messe comme une actualisation de la célébration unique } \\
& \text { La Cène de Jésus-Christ: } \\
& \text { Quand donc vous vous réunissez dans un même lieu, ce n'est pas la cène du } \\
& \text { Seigneur que vous mangez. Car, au moment de manger, chacun s'empresse de } \\
& \text { prendre son propre repas, et l'un a faim, et l'autre s'enivre. N'avez-vous donc pas } \\
& \text { des maisons pour manger et pour boire ? Ou méprisez-vous l'Assemblée de Dieu et } \\
& \text { faites-vous honte à ceux qui n'ont rien? Que vous dirai-je? Vous louerai-je? En } \\
& \text { cela, je ne vous loue pas. Car moi, j'ai reçu du Seigneur ce qu'aussi je vous ai } \\
& \text { enseigné, c'est que le Seigneur Jésus, la nuit où il fut livré, prit du pain, et après } \\
& \text { avoir rendu grâce, il le rompit et dit: «Ceci est mon corps qui est pour vous; faites } \\
& \text { ceci en mémoire de moi. » De même [il prit] aussi la coupe après le dîner, en disant: } \\
& \text { "Cette coupe est la nouvelle alliance en mon sang; faites ceci toutes les fois que } \\
& \text { vous la boirez, en mémoire de moi.» (Première Épître aux Corinthiens 11, 23-26) }
\end{aligned}
$$

Dans le discours de Jésus, nous pouvons comprendre à quel point la symbolique du vin est riche. Et les mots qu'il prononce lors de la Cène nous font deviner des images de la joie, du passage de la vie à la mort, du sacrifice, de la Résurrection et du banquet avec 
Dieu. Ce dernier est, d'ailleurs, bien explicite dans ce passage de la Bible. Jésus annonce qu'il ne boira du vin nouveau que dans le royaume de Dieu :

Et comme ils mangeaient, Jésus, ayant pris du pain [et] ayant béni, le rompit, et [le] leur donna, et dit: «Prenez, ceci est mon corps.» Puis, ayant pris une coupe [et] ayant rendu grâce, il [la] leur donna et ils en burent tous. Et il leur dit : "Ceci est mon sang, le [sang] de l'alliance, qui est versé pour un grand nombre. En vérité, je vous dis que je ne boirai plus du tout du fruit de la vigne jusqu'à ce jour où je le boirai nouveau dans le royaume de Dieu. » Et ayant chanté des hymnes, ils sortirent [pour aller] au mont des Oliviers. (Marc 14, 22-26)

Bénédiction divine, la Cène de Jésus-Christ est de l'ordre du transcendantal. Simple mais fiable, elle propose la célébration de l'Éternel. C'est, avant tout autre chose, faire vivre le sacré divin. Et les Noces de Cana? Faute humaine, le manque de vin souligne la faille humaine. Vivre les noces, c'est dire l'éphémère, c'est déclarer la faute, c'est professer la célébration de la vie. C'est, avant toute autre chose, faire vivre le profane humain. La Cène et les noces de Cana sont les deux modèles de repas qui représentent, encore de nos jours, la différence entre le sacré et le profane.

En somme, en revisitant quelques exemples de la Bible, nous avons compris à quel point les métaphores et les paraboles autour du vin deviennent «toutes puissantes ». À usage médical ou domestique, signe de joie ou de sexualité, le vin devient surtout symbole de vie et de bénédiction de Dieu. Nous avons, donc, analysé quelques-uns des aspects positifs - parce que la vigne est signe de richesse et de bénédiction divine - et des aspects négatifs - car l'ivresse est envisagée comme faute. Mais, que ce soit une faute humaine ou une bénédiction divine, en tournant les pages de la Bible, nous avons été surpris par le nombre infini de références autour de la vigne et du vin. En fait, dans la Bible ce breuvage, agréable à boire, est associé à une multitude de métaphores et de paraboles.

En guise de conclusion, nous pouvons affirmer que le vin est signe de bonheur, de vie et suivre le conseil biblique : «Alors, mange ton pain avec plaisir et bois ton vin d'un cœur joyeux, car Dieu a déjà approuvé tes actions. » (Ecclésiastes, 9,7)

\section{BIBLIOGRAPHIE}

CARREIRA DAS NEVES, Joaquim. (1984). “A redenção à luz das narrativas da Instituição da Eucaristia”, Didaskalia, nº XIV, pp. 113-137.

CAMBRONNE, Patrice (2007). " La tradition des Écritures sacrées : Christianisme, Judaïsme, Islam », in Françoise Argod-Dutard, Pascal Charvet et Sandrine Lavaud (orgs.). Voyages au pays du vin, Paris : Robert Laffont, pp. 55-132.

CENTRE BIBLIQUe (2021). « Bible en Ligne » [on-line]. France : Centre Biblique [disponible le 07/04/2021] <URL : http://www.bibleenligne.com/la-bible.html>.

Delgado, José Fernando. (1994). « Los moralistas y la alimentício em la Baja Eda Media », História 16, no 233 , pp. 47-66. 
HOMÈRE. (2018). L'Odyssée. (Trad.) Victor Bérard. Paris : Les Belles Lettres.

LAGRANGE, Marc (2004). Le Vin \& la Médecine. À l'usage des bons vivants et des médecins. Bordeaux :

Éditions Féret.

LORCIN, Marie-Thérèse (1998). « Les usages du vin à la fin du Moyen Âge » in Pour l'aise du corps.

Paris : Paradigma, pp. 261-273.

REFFóIOS, Margarida (2010). Saber e Sabores - Aspetos da Cultura Alimentar Europeia. Caleidoscópio :

Casal de Cambras.

\section{RÉSUMÉS}

Le champ de signification de la vigne et du vin est passionnant. L'imaginaire du vin emprunte à la culture christique une symbolique qui fait vivre in aeternum le pouvoir de la religion. En revisitant quelques exemples de la Bible, nous essayerons de comprendre à quel point les métaphores et les paraboles autour du vin deviennent «toutes puissantes ». À usage médical ou domestique, signe de joie ou de sexualité, le vin devient surtout symbole de vie et bénédiction de Dieu. Nous nous attacherons à en dégager les aspects positifs - parce que la vigne apparaît comme signe de richesse et de bénédiction divine - et les aspects négatifs - car l'ivresse est envisagée comme faute.

The significance field of the vine and wine is extraordinary. The imaginary of wine borrows from the Christian culture a symbolism that makes the power of religion live in aeternum. By revisiting some examples of the Bible, we will try to understand how metaphors and parables around wine become «all powerful ». For medical or domestic use, a sign of joy or sexuality, wine becomes above all a symbol of God's life and blessing. In short, we will emphasize above all the positive aspects - because the vine appears as a sign of wealth and divine blessing - and the negative aspects - because drunkenness is considered as a fault.

\section{INDEX}

Keywords : wine, health, religion, imaginary, Bible

Mots-clés : vin, santé, religion, imaginaire, Bible

\section{AUTEUR}

MARGARIDA PINA

NOVA FCSH - IELT

mepreffoios[at]fcsh.unl.pt 